\title{
Formation of Complement Membrane Attack Complex in Mammalian Cerebral Cortex Evokes Seizures and Neurodegeneration
}

\author{
Zhi-Qi Xiong, ${ }^{1}$ Weihua Qian, ${ }^{1}$ Katsuaki Suzuki, ${ }^{1}$ and James 0. McNamara ${ }^{1,2,3,4}$ \\ Departments of ${ }^{1}$ Neurobiology, ${ }^{2}$ Medicine (Neurology), and ${ }^{3}$ Pharmacology and Molecular Cancer Biology, Duke University Medical Center, Durham, \\ North Carolina 27710, and ${ }^{4}$ Durham Veterans Affairs Medical Center, Durham, North Carolina 27710
}

\begin{abstract}
The complement system consists of $>30$ proteins that interact in a carefully regulated manner to destroy invading bacteria and prevent the deposition of immune complexes in normal tissue. This complex system can be activated by diverse mechanisms proceeding through distinct pathways, yet all converge on a final common pathway in which five proteins assemble into a multimolecular complex, the membrane attack complex (MAC). The MAC inserts into cell membranes to form a functional pore, resulting in ion flux and ultimately osmotic lysis. Immunohistochemical evidence of the MAC decorating neurons in cortical gray matter has been identified in multiple CNS diseases, yet the deleterious consequences, if any, of MAC deposition in the cortex of mammalian brain in vivo are unknown. Here we demonstrate that the sequential infusion of individual proteins of the membrane attack pathway (C5b6, C7, C8, and C9) into the hippocampus of awake, freely moving rats induced both behavioral and electrographic seizures as well as cytotoxicity. The onset of seizures occurred during or shortly after the infusion of $\mathrm{C} 8 / \mathrm{C} 9$. Neither seizures nor cytotoxicity resulted from the simultaneous infusion of all five proteins premixed in vitro. The requirement for the sequential infusion of all five proteins together with the temporal relationship of seizure onset to infusions of $\mathrm{C} 8 / \mathrm{C} 9$ implies that the $\mathrm{MAC}$ was formed in vivo and triggered both seizures and cytotoxicity. Deposition of the complement MAC in cortical gray matter may contribute to epileptic seizures and cell death in diverse diseases of the human brain.
\end{abstract}

Key words: complement; membrane attack complex; C5b9; seizure; neurodegeneration; hippocampus; Fluoro-Jade B

Complement is an important member of the innate immune system and also one of the major effector mechanisms of the humoral component of the adaptive immune system (Walport, 2001). Although diverse mechanisms can activate complement, each activation pathway culminates in the formation of $\mathrm{C} 5 \mathrm{~b}$, the first component of the membrane attack pathway. Once formed, C5b binds to C6 to produce a stable and soluble complex, C5b6. Next, C7 binds C5b6 to form C5b7, which can attach to the surface of cell membranes without disturbing membrane integrity. The binding of $\mathrm{C} 8$ to the membrane-bound C5b7 forms C5b8, which becomes more deeply incorporated in the membrane and causes the cell to become slightly leaky. The C5b8 complex in turn forms a receptor for $\mathrm{C} 9$ molecules. The binding of the initial C9 molecule to C5b8 transforms the C9 molecule from a globular, hydrophilic structure to an elongated, amphipathic structure, which inserts into and through the membrane; these conformational changes in C9 expose binding sites for additional C9 to bind, unfold, and insert into the membrane. Addition of as many as 18 copies of $\mathrm{C} 9$ to the C5b8 complex forms the membrane attack complex (MAC), resulting in ion flux and ultimately lysis of target cells (Morgan, 1999).

\footnotetext{
Received Sept. 20, 2002; revised Nov. 4, 2002; accepted Nov. 8, 2002.

This work was supported by National Institute of Neurological Disorders and Stroke Grant NS3038319. We thank Dr. Steve Danzer for his comments on this manuscript.

Correspondence should be addressed to Dr. James 0 . McNamara, Department of Neurobiology, Box 3676, Duke University Medical Center, Durham, NC27710.E-mail:jmc@neuro.duke.edu.

K. Suzuki's present address: Department of Psychiatry and Neurology, Hamamatsu University School of Medicine, Hamamatsu, Shizuoka 431-3192, Japan.

Copyright $\odot 2003$ Society for Neuroscience $\quad 0270-6474 / 03 / 230955-06 \$ 15.00 / 0$
}

Although its normal function is critical to the health of the organism, inappropriate activation of complement can itself damage tissue of multiple organs and contribute to disease in humans (Morgan, 1994; Matis and Rollins, 1995; Morgan, 1995; Asghar and Pasch, 2000). With respect to diseases of the CNS, inappropriate activation of complement appears to contribute to demyelination in multiple sclerosis. Evidence of inappropriate activation of complement in multiple sclerosis includes MAC immunoreactivity in myelin (Compston et al., 1989) together with MAC immunoreactive membranes isolated from the spinal fluid of patients with multiple sclerosis (Scolding et al., 1989). Moreover, transgenic overexpression of a complement inhibitory protein eliminates demyelination in an animal model of multiple sclerosis (Davoust et al., 1999), underscoring the deleterious effects of complement activation in this disease. A wealth of immunohistochemical evidence has demonstrated inappropriate activation of complement, including the formation of MAC, on neurons in cortical gray matter in diverse neurodegenerative diseases, including Alzheimer's (McGeer et al., 1989; Itagaki et al., 1994; Webster et al., 1997; Yasojima et al., 1999), Huntington's (Singhrao et al., 1999), and Pick's (Yasuhara et al., 1994) diseases, as well as in the putative autoimmune disease Rasmussen's encephalitis (Whitney et al., 1999). The evidence of MAC formation on cortical neurons in these diverse diseases notwithstanding, whether the deposition of MAC in cortical gray matter in the mammalian brain has deleterious consequences is uncertain. The determination that the formation of MAC in muscle cell membranes results in depolarization (Jackson et al., 1981) together with the cytotoxic effects of MAC in cortical neurons in vitro 
(Whitney and McNamara, 2000) led us to hypothesize that MAC deposition on neurons could trigger seizures and neurodegeneration. We tested this hypothesis by sequential infusion of individual proteins of the membrane attack pathway into the rat hippocampus to form MAC in vivo.

\section{Materials and Methods}

Electrode implantation and intrahippocampal cannulation. Male Sprague Dawley rats (250-300 gm) were anesthetized with sodium pentobarbital $(60 \mathrm{mg} / \mathrm{kg})$ and placed in a stereotaxic frame. Teflon-coated stainlesssteel bipolar electrodes were implanted into the left dorsal hippocampus (the bregma was used as the reference; coordinates: $-3.5 \mathrm{~mm}$ anteroposterior, $+2.5 \mathrm{~mm}$ lateral, $-3.2 \mathrm{~mm}$ ventral to the dura) (Paxinos and Watson, 1982). The tip of a guide cannula (26 gauge; Plastics One, Roanoke, VA) was placed into the contralateral (right) dorsal hippocampus $(-3.5 \mathrm{~mm}$ anteroposterior, $+2.5 \mathrm{~mm}$ lateral, and $-2.5 \mathrm{~mm}$ ventral to the dura). The cannula and electrode were secured firmly to the skull with dental acrylic and a ground wire was attached to an anchor screw (see Fig. 1A). After surgery, a removable stylet was placed in the guide cannula and the animals were allowed to recover for $4 \mathrm{~d}$. The EEG is recorded from the hippocampus contralateral to the injected hippocampus, because the rich commissural connections facilitate seizure propagation between the two hippocampi, thereby simplifying the detection of electrographic seizure; also any damage resulting from the placement of the recording electrode will not confound the assessment of damage caused by the infusion of complement.

Intrahippocampal infusion and EEG recording. On the day of infusion, the stylet was removed and an infusion cannula (28 gauge) was inserted through the guide cannula to a depth of $3.5 \mathrm{~mm}$ below the dura. Solutions were infused into the hippocampus of awake, freely moving rats at a rate of $0.20 \mu \mathrm{l} / \mathrm{min}$, and EEG and behavior were continuously monitored. The intensity of behavioral seizures was scored according to a modification of Racine (1972), as follows: class 1, facial clonus and chewing; class 2, head nodding; class 3, unilateral forelimb clonus; class 4, rearing with bilateral forelimb clonus; class 5 , rearing and falling (loss of postural control); class 6 , running; class 7 , running, jumping, and tonicclonic seizure.

Perfusion. Animals were killed at varying intervals after infusion by deep anesthesia (pentobarbital, $60 \mathrm{mg} / \mathrm{kg}$, i.p.) followed by perfusion with $100 \mathrm{ml}$ of cold saline and subsequently with $250 \mathrm{ml}$ of $0.1 \mathrm{M}$ sodium phosphate-buffered ( $\mathrm{pH} 7.4$ ) paraformaldehyde (4\%). After additional fixation overnight in buffered paraformaldehyde, the brains were cryopreserved for $48 \mathrm{hr}$ in $20 \%$ sucrose in phosphate buffer and frozen in isopentene chilled in a dry ice-methanol bath. Coronal sections $(40 \mu \mathrm{m})$ were collected in 24 well culture plates containing PBS or mounted onto gelatin-coated slides and stored at $4^{\circ} \mathrm{C}$ until use.

$M A C$ immunohistochemistry. Floating sections were washed in PBS, incubated for $1 \mathrm{hr}$ in blocking buffer (PBS containing 0.1\% Triton X-100 and $2 \%$ bovine serum albumin), and then incubated with rabbit antihuman C5b9 in blocking buffer (final concentration, $1 \mu \mathrm{g} / \mathrm{ml}$; Calbiochem, La Jolla, CA) for $1 \mathrm{hr}$ at room temperature. After three washes, sections were incubated for $1 \mathrm{hr}$ in biotinylated goat anti-rabbit IgG ( 1.5 $\mu \mathrm{g} / \mathrm{ml}$ ) (Jackson ImmunoResearch Laboratories, West Grove, PA) diluted in blocking buffer (PBS containing $0.1 \%$ Triton X-100 and $5 \%$ goat serum). To visualize biotinylated secondary antibodies, cultures were washed three times in PBS, followed by a 30 min incubation with streptavidin-linked rhodamine (final concentration $4 \mu \mathrm{g} / \mathrm{ml}$; Molecular Probes, Eugene, OR) in PBS. After three washes, the sections were dried, mounted, and viewed with a microscope equipped for epifluorescence.

Nissl staining. Sections were mounted from $0.1 \mathrm{~m}$ sodium phosphate buffer, $\mathrm{pH}$ 7.4, onto gelatin-coated slides and dried at room temperature. After rinsing with tap water, sections were stained for $5 \mathrm{~min}$ in $0.5 \%$ toluidine blue. After graded alcohol washes, the inserts were briefly dipped in $95 \%$ ethanol containing $1 \%$ glacial acetic acid. Sections were then rehydrated and mounted.

Fluoro-Jade B staining. Degenerating neurons were detected with Fluoro-Jade B as described by Schmued and Hopkins (2000). Briefly, sections were mounted from $0.1 \mathrm{~m}$ sodium phosphate buffer, $\mathrm{pH} 7.4$,
A

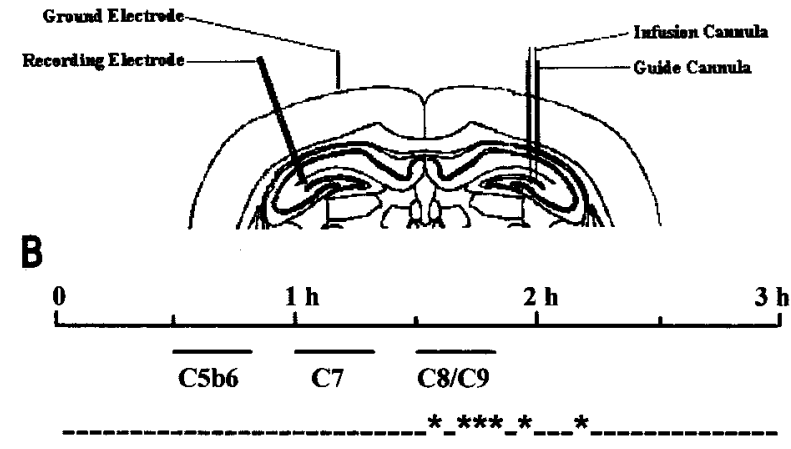

C
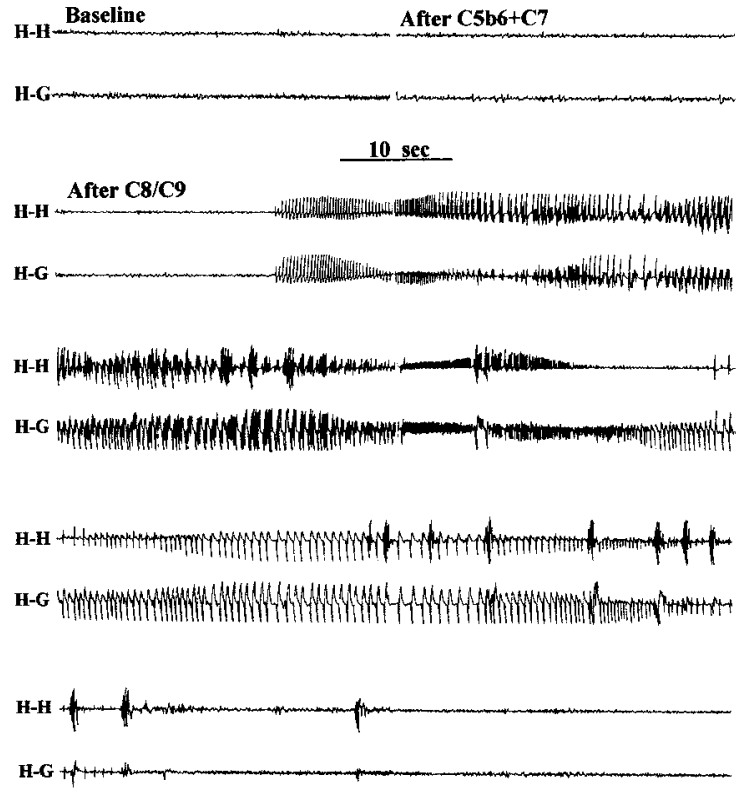

Figure 1. Formation of MAC in rat brain evokes seizures. $A$, Placements of recording electrode and infusion cannula inserted through the guide cannula. $B$, Timeline of experiments in which complement proteins are infused into the right hippocampus of awake, freely moving rats (top), and the occurrence of seizures detected in EEG recordings from a representative animal (bottom; asterisks indicate seizure events). C, EEG recordings between the tips of bipolar electrodes in hippocampus $(H-H)$ or from one of these electrodes referenced to a skull screw $(H-G)$ from a representative animal in which the infusion of $(5 b 9$ induced seizures. This is the sixth seizure in this animal.

onto gelatin-coated slides, dried, and immersed in $100 \%$ ethanol for $3 \mathrm{~min}$, followed by $70 \%$ ethanol for $5 \mathrm{~min}$ and then distilled water for $2 \mathrm{~min}$; they were then incubated in $0.06 \%$ potassium permanganate for $15 \mathrm{~min}$. After rinsing with distilled water, the slides were incubated in $0.001 \%$ Fluoro-Jade B (Histo-Chem, Inc., Jefferson, AR) in $0.1 \%$ acetic acid for $25 \mathrm{~min}$, rinsed in water three times, dried at room temperature, dehydrated in xylene, and coverslipped with DPX (Electron Microscopy Sciences, Fort Washington, PA).

\section{Results}

To induce the formation of MAC in mammalian cerebral cortex, purified complement proteins (Advanced Research Technologies, San Diego, CA) of the membrane attack pathway were infused sequentially (Fig. $1 B$ ) through a guide cannula in the right hippocampus of awake freely moving rats while continuously monitoring behavior; the EEG was recorded from the contralateral hippocampus (Fig. 1A). A representative experiment (Fig. $1 B, C$ ) revealed a normal EEG before the infusion (baseline) and 
during and after the infusion ( $3 \mathrm{nmol}$ ) of C5b6, after which the injection cannula was withdrawn and replaced with another injection cannula through which C7 (4 nmol) was infused. A normal EEG was also recorded during and after the infusion of C7. At this point, the injection cannula was again withdrawn and replaced with a third injection cannula through which a solution containing C8 (5 nmol) and C9 (14 nmol) was infused. Six electrographic seizures were observed at the times denoted by asterisks in Figure $1 \mathrm{~B}$. The first seizure occurred $5 \mathrm{~min}$ after initiating the infusion of C8/C9 and lasted $280 \mathrm{sec}$ (data not shown). The sixth seizure (Fig. 1C) occurred $20 \mathrm{~min}$ after terminating the infusion of C8/9 and lasted for $160 \mathrm{sec}$, during which the animal exhibited clonic contractions of the forelimbs typical of class 3 and class 4 seizures. No additional seizures were detected despite continuous monitoring for at least $1 \mathrm{hr}$ and a brief monitoring session $(30 \mathrm{~min})$ the next day. In these initial experiments, seizures (three to six per animal) were observed in each of three animals infused with these doses, yet no seizures were observed in three control animals in which artificial CSF (ACSF) was substituted for C5b6, C7, or C8/C9. Likewise no seizures were observed in either of two additional controls infused with "preformed C5b9," demonstrating that simple infusion of five proteins is not sufficient to induce seizures; that is, all five proteins were mixed in vitro, resulting in the formation of the C5b9 complex in solution, after which the complexes would not be expected to insert into a cell membrane (Morgan, 1999). The temporal relationship of seizure onset with the introduction of $\mathrm{C} 8 / 9$, together with the fact that seizures were evoked by $\mathrm{C} 8 / 9$ only in animals with previous infusion of C5b6 and C7, implies that the MAC was formed in vivo and triggered the seizures.

The fact that assembly of the MAC can be limited by the amount of $\mathrm{C} 5 \mathrm{~b}$ generated by the early activation pathway of the complement cascade suggested a strategy with which to establish a dose-response curve. Varying amounts of C5b6 were infused, followed by a fixed, and presumably molar, excess amount of $\mathrm{C} 7$ and $\mathrm{C} 8 / \mathrm{C} 9$, which should thereby vary the density of MAC formed in the tissue. The doses were plotted as amounts (in nanomoles) of C5b6 infused, and the responses were plotted as the percentage of animals exhibiting seizures (Fig. $2 A$ ), behavioral intensity, or the number or duration of seizures (Fig. $2 B-D$ ). The highest dose ( $3 \mathrm{nmol}$ of C5b6) evoked seizures in 10 of 11 rats, resulting in an average of five seizures per animal with an average behavioral intensity of class 4 . The effects were dose dependent, as evidenced by fewer seizures of shorter duration and diminished behavioral intensity with the doses of 1.5 and $0.75 \mathrm{nmol}$ of C5b6.

To determine whether MAC formation also caused cytotoxicity, animals from the experiments described in the preceding paragraph were killed $2 \mathrm{~d}$ after infusion for histological study. In contrast to the normal hippocampal architecture of the recording (noninfused) side (Fig. 3A,B, left), extensive destruction of each of the principal populations of hippocampal neurons is evident in Nissl stains of the hippocampus infused with $3 \mathrm{nmol}$ of C5b6 followed by C7/C8/C9 (Fig. 3A,B, right top). Fluoro-Jade B staining of nearby sections revealed degenerating neurons in the infused but not the contralateral hippocampus (Fig. 3A, B, compare bottom right and bottom left). Infusion of a lower dose of C5b6 $(0.75 \mathrm{nmol})$ produced less cell loss, as evidenced by a representative animal in which destruction was confined to the dentate gyrus, hilus, and CA4 area near the site of the infusion (Fig. $3 C, D)$. The destruction evident on Nissl and Fluoro-Jade B staining was not simply a consequence of the infusion, because controls in which ACSF was substituted for C5b6, C7, or C8/C9 exhibited negligible cell death (data not shown). Likewise, small
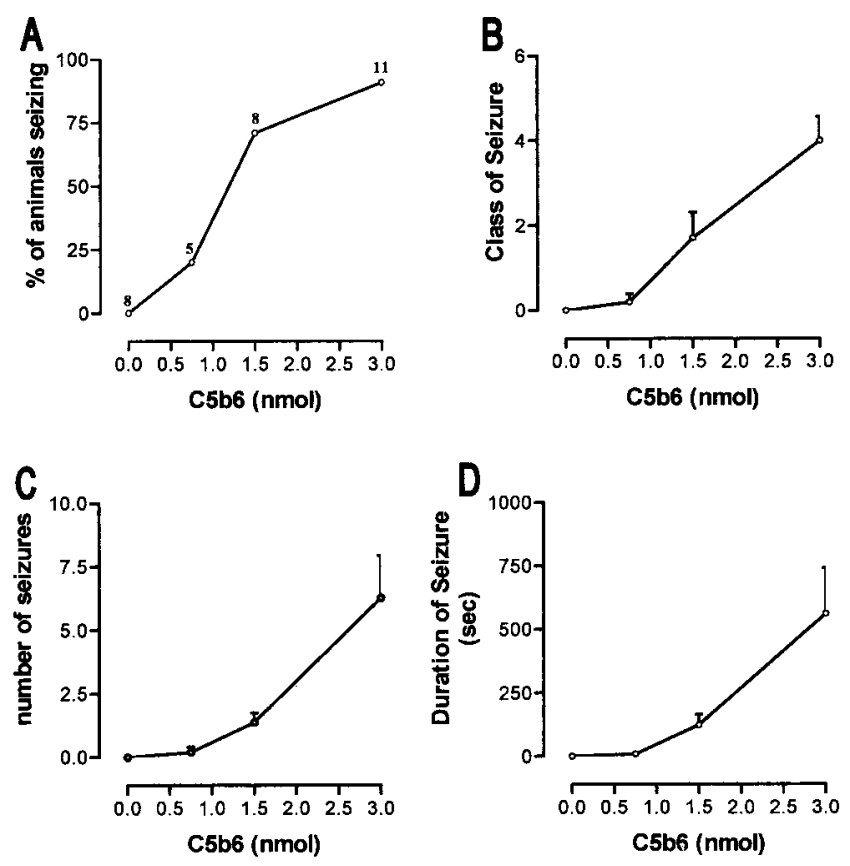

Figure 2. Concentration dependence of effects of $C 5 \mathrm{~b} 6$ in the presence of excess $C 7 / C 8 / C 9$ on seizure parameters. $A$, Effects of varying amounts of $C 5 b 6$ followed by $C 7, C 8$, and $C 9$ on the percentage of animals exhibiting seizures. The number of animals receiving each amount of C5b6 is shown above the data points. The results presented in this figure were obtained from animals described in Results as well as from additional animals studied to confirm and extend the findings of the initial experiments. $B-D$, Results of experiments presented in $A$ are presented as seizure severity $(B)$, the number of seizures per animal $(C)$, and the total duration of seizure activity per animal during the recording period $(D)$. Error bars indicate SEM.

inflammatory cell infiltrates but minimal or no cytotoxicity were evident in animals infused with preformed C5b9, as evidenced by the representative animal shown in Figure 3E,F. The dose dependence of the cytotoxicity was also evident in the semiquantitative measures of destruction of the three principal neuronal populations of hippocampus (Fig. 4).

To further assess MAC formation in vivo, immunohistochemical experiments were performed. In these experiments, five additional animals underwent the sequential infusion of C5b6 (3 $\mathrm{nmol}$ ), followed by $\mathrm{C} 7$ and finally $\mathrm{C} 8 / \mathrm{C} 9$, using the highest dose shown in Figure $2 A-D$; in contrast to previous experiments, these animals were killed $1 \mathrm{hr}$ after the infusion. The interval of just $1 \mathrm{hr}$ between the infusion and death was selected because the occurrence of seizures during and shortly after the infusion of $\mathrm{C} 8 / \mathrm{C} 9$ implied that the MAC was formed during or shortly after the infusion and thus should be detectable at this time. Seizures were observed in each of the five animals infused. In the animal presented in Figure $5 A, B$, intense MAC immunoreactivity was evident in the hippocampus sequentially infused with C5b6, C7, and C8/C9 (right); in contrast, no MAC immunoreactivity was detected in the contralateral hippocampus from which the electrographic seizure activity was recorded (Fig. 5A, B, left). The immunoreactivity was widespread within, but did not extend beyond, the infused hippocampus (Fig. $5 A, B$, right). Elimination of the immunoreactivity by preincubating the primary antibody with soluble C5b9 (Fig. 5C) reinforces the specificity of the antibody. The occurrence of seizures temporally related to the infusion of C8/C9 implies that the MAC was formed in all five animals, yet MAC immunoreactivity was detected in only three of the five animals infused. The most likely explanation for the absence of detectable MAC immunoreactivity in the remaining two animals 

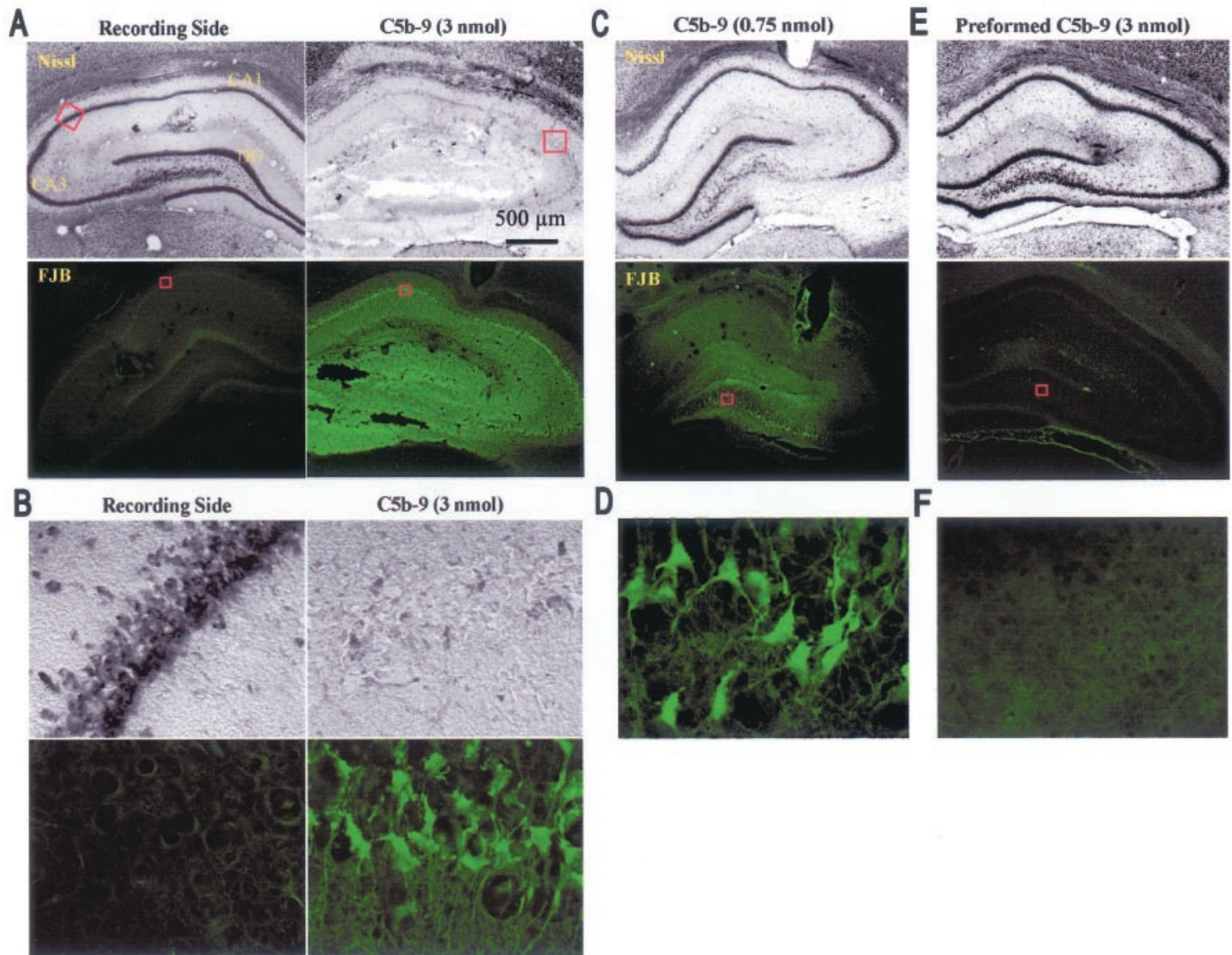

Figure 3. Formation of $M A C$ in rat brain induces neurodegeneration. $A$, Nissl (top) and Fluoro-Jade $B$ ( $F J B$; bottom) staining of rat brain sections after the sequential infusion of $C 5 b 6$ ( $3 \mathrm{nmol})$, $C 7$ $(4 \mathrm{nmol})$, and $(8(5 \mathrm{nmol}) / \mathrm{C} 9(14 \mathrm{nmol})$ into the right hippocampus of the rat. There is massive cell death on the injected side but not on the recording side. B, High-power photomicrograph of the areas designated by boxes in $A$ disclosed the loss of CA1 pyramidal neurons in Nissl staining (top right) and the presence of Fluoro-Jade B labeling of degenerating cell bodies and proximal dendrites of CA1 pyramidal neurons (bottom right). C, Nissl (top) and Fluoro-Jade B (bottom) staining of rat hippocampus after the sequential infusion of lower amounts $(0.75 \mathrm{nmol})$ of $C 5 \mathrm{~b} 6$ in the presence of excess $\mathrm{C} 7 / C 8 / C 9$. Cell loss and Fluoro-Jade-B-positive cells were confined to the injected area, including the granule cell layer, hilar neurons, and CA4 pyramidal cells. D, High-power photomicrograph of the boxed area of ( disclosed the presence of Fluoro-jade B labeling of degenerating cell bodies and proximal dendrites of CA4 pyramidal neurons. E, NissI (top) and Fluoro-Jade B (bottom) staining of rat hippocampus after the infusion of preformed $3 \mathrm{nmol}$ C5b9. There is no obvious cell loss, but there are minimal inflammatory cell infiltrates in the injected area evident on Nissl staining. $F$, High-power photomicrograph of the boxed area of $E$. DG, Dentate gyrus.

is that the epitope was destroyed by proteolytic enzymes during the hour after the infusion; the fact that no additional seizures occurred later than $30 \mathrm{~min}$ after terminating the infusion of $\mathrm{C} 8 / \mathrm{C} 9$ suggests that functional MACs are rapidly eliminated and thus could have escaped detection by immunohistochemistry. Other potential explanations include inaccessibility of the epitope to the antibody, as found by Morgan et al. (1987), or the presence of MAC in a section of hippocampus not selected for immunohistochemical study.

\section{Discussion}

The principal findings emerging from these studies include the following. Sequential infusion of the five proteins of the membrane attack pathway into the hippocampus of awake, freely moving rats induced both behavioral and electrographic seizures as well as cytotoxicity. The onset of seizures occurred during or shortly after the infusion of $\mathrm{C} 8 / \mathrm{C} 9$. In contrast, the simultaneous infusion of all five proteins premixed in vitro evoked neither seizures nor cytotoxicity. Likewise, the sequential infusion of just three or four of the five proteins of the membrane attack pathway evoked neither seizures nor cytotoxicity. The requirement for the sequential infusion of all five proteins together with the temporal relationship of seizure onset to infusions of $\mathrm{C} 8 / \mathrm{C} 9$ implies that the MAC was formed in the infused hippocampus in vivo and triggered both seizures and cytotoxicity. The presence of MAC immunoreactivity in a subset of animals undergoing infusion reinforces this conclusion.

Studies of MAC effects in both excitable and nonexcitable cells in vitro provide a clue to the mechanism by which MAC deposition on neurons in vivo might trigger seizures. The fully assembled MAC contains one molecule each of C5b, C6, C7, and C8 and multiple C9 molecules; insertion of MACs into cell membranes creates transmembrane channels. Single-channel analyses of MACs formed in planar lipid bilayers by the sequential application of purified human complement proteins, C5b6, C7, C8, and C9 (Benz et al., 1986) revealed striking variation of the singlechannel conductances, the largest of which were $3.1 \mathrm{nS}$. The channels exhibited linear current-voltage relationships and, interestingly, a threefold to fourfold greater permeability for cations compared with anions. Indeed, MAC formation in erythrocyte membranes leads to the influx of $\mathrm{Ca}^{2+}$ and $\mathrm{Na}^{+}$and the efflux of $\mathrm{K}^{+}$(Halperin et al., 1993); the net effect is a dosedependent depolarization of membrane potential before hemolysis (Wiedmer and Sims, 1985). MAC deposition on the membrane of cardiac myocytes induced increases in intracellular $\mathrm{Ca}^{2+}$ 


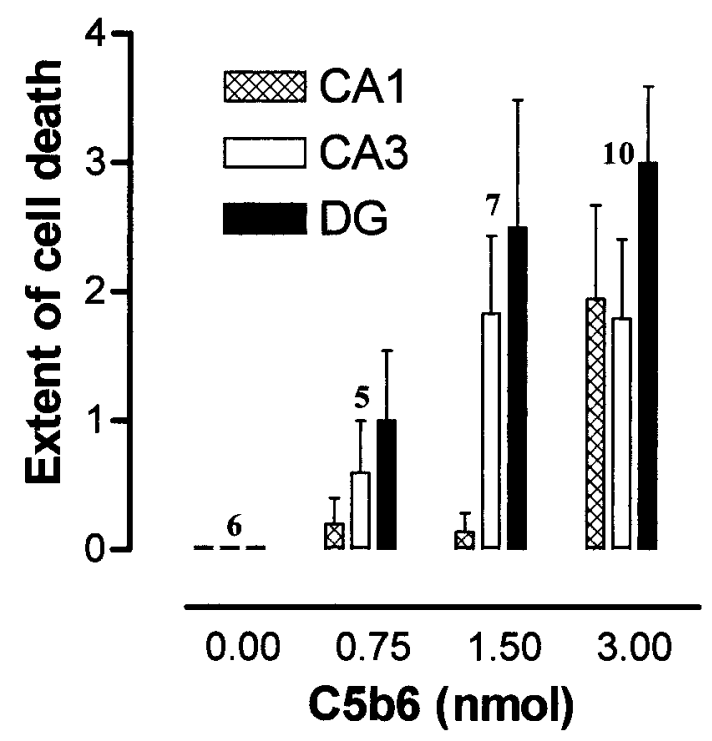

Figure 4. Concentration dependence of effects of $C 5 \mathrm{~b} 6$ in the presence of excess $\mathrm{C} / \mathrm{C} 8 / \mathrm{C} 9 \mathrm{on}$ neuronal cell death. The intensity of cell death was graded on a semiquantitative scale ranging from 0 to 5 . The numbers of animals included in these histological analyses are specified above the CA3 columns and represent a subset of the animals presented in Figure 2; the remaining animals were used for different histological studies, the results of which are not presented here. Note that cell death was evident in the hippocampus of all animals exhibiting seizures; however, some cell death was also detected in the injected hippocampus after the infusion of complement in which seizures were not detected. DG, Dentate gyrus.

and contractility (Berger et al., 1993). Thus, it seems plausible that the deposition of MACs on the membranes of a small subset of hippocampal neurons may trigger similar cation fluxes and depolarization; the resulting high-frequency firing of this subset might recruit otherwise unaffected but synaptically coupled neurons into a larger neuronal population firing synchronously, thus mediating the electrographic and behavioral seizures observed in this study. Similar mechanisms might underlie the medically refractory seizures commonly observed in Rasmussen's encephalitis, in which MAC deposition on cortical neurons has been identified immunohistochemically (Whitney et al., 1999).

Mechanisms similar to those by which MAC deposition evokes seizures likely underlie the cytotoxic consequences of MACs in the present study. Although the deposition of one or a few MACs might evoke cation flux and depolarization, triggering high-frequency firing, the diffusion of ions down their electrochemical gradients through large numbers of MACs would be expected to induce cell swelling and osmotic lysis, the hallmarks of death of cortical neurons undergoing lethal attack by complement in vitro (Whitney and McNamara, 2000). Importantly, analyses of diverse nucleated cell types have elucidated an elaborate set of defense mechanisms aimed at fending off MAC deposition on cell membranes. An early defense is mediated by CD59, a cell-surface complement regulatory protein, which binds nascent $\mathrm{C} 5 \mathrm{~b} 9$ complexes and prevents the incorporation of additional C9 molecules and formation of a full MAC (Morgan, 1999). If this defense is overwhelmed and MACs are deposited in the cell membrane, the cell eliminates the membrane fragments containing the MACs by both endocytosis and exocytosis (Morgan et al., 1987; Scolding et al., 1989; Stein and Luzio, 1991), simultaneously increasing ion pumping to restore ionic homeostasis. In the present experiments, the rapid infusion of high concentrations of proteins of the membrane attack pathway presumably overwhelmed the defense mechanisms and effected

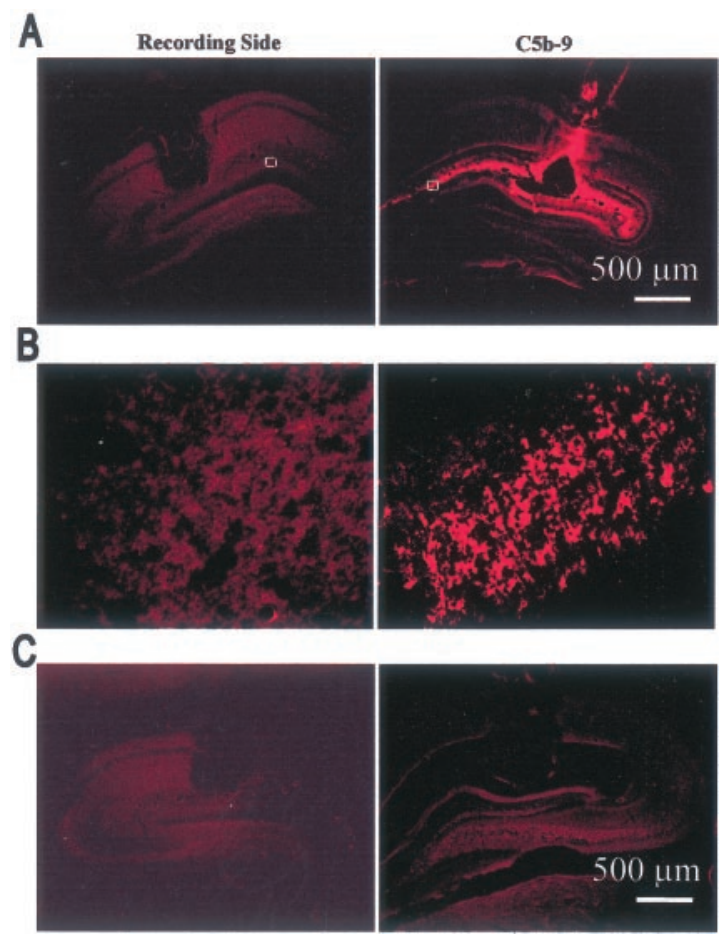

Figure 5. MAC immunohistochemistry after intrahippocampal infusion of complement proteins. $A$, MAC immunoreactivity in the hippocampus of an animal killed $1 \mathrm{hr}$ after the sequential infusion of ( $5 \mathrm{~b} 6$ ( $3 \mathrm{nmol}), \mathrm{C} 7(4 \mathrm{nmol})$, and $(8$ ( $5 \mathrm{nmol}) / C 9(14 \mathrm{nmol})$ into the right hippocampus (right). Note the absence of detectable MAC immunoreactivity in the uninjected hippocampus (left) used for recording of the same animal. $B$, High-power photomicrograph of the boxed areas in $A$. C, The MAC immunoreactivity was eliminated when the anti-MAC antibodies were preincubated with soluble $C 5 \mathrm{~b} 9$ in sections adjacent to those portrayed in $A$, demonstrating the antibody specificity.

rapid necrotic death of neurons, as evidenced both by Nissl and Fluoro-Jade B staining.

Although the present studies elucidate some deleterious consequences of MAC deposition in cortical gray matter in vivo, the complement cascade almost certainly has both adaptive and maladaptive consequences in a number of pathological settings. One common disease exemplifying this idea is Alzheimer's disease, in which the accumulation of extracellular $A \beta$ peptide contributes to the pathogenesis (Hardy and Selkoe, 2002). Aggregated A $\beta$ peptide can activate both the classical and alternative complement cascades in vitro by binding $\mathrm{C} 1 \mathrm{q}$ and $\mathrm{C} 3 \mathrm{~b}$, respectively (Jiang et al., 1994; Bradt et al., 1998); this is likely to be the trigger of sequential activation of these pathways, resulting in the formation of C5b, the initial component of the membrane attack pathway, thereby leading to the deposition of the MAC evident in Alzheimer's disease brains (McGeer et al., 1989; Itagaki et al., 1994; Webster et al., 1997; Yasojima et al., 1999). Interestingly, the binding of $\mathrm{A} \beta$ peptide by $\mathrm{C} 3 \mathrm{~b}$ likely promotes its phagocytosis and removal, because the transgenic overexpression of an inhibitor of $\mathrm{C} 3 \mathrm{~b}$ formation increases $\mathrm{A} \beta$ peptide accumulation in mice expressing the human amyloid precursor protein (WyssCoray et al., 2002); importantly, the increased $\mathrm{A} \beta$ peptide deposition is accompanied by enhanced neurodegeneration in these mice (Wyss-Coray et al., 2002). These adaptive consequences notwithstanding, the triggering of the early activation pathway of complement by $\mathrm{A} \beta$ peptide leaves the complement cascade poised to transition to the membrane attack pathway and deposition of the MAC. Notably, the transition to the membrane attack pathway is not an inevitable consequence of triggering the 
early activation pathway; rather, a diversity of complement regulatory proteins expressed on neurons and other cell types inhibits diverse steps in the complement cascade, seeking to limit the deleterious consequences of complement activation on host cells (Liszewski et al., 1996). Immunohistochemical evidence of MAC deposition on neurons in Alzheimer's brains implies that the endogenous defenses are not completely effective. Interestingly, supervening sublethal excitotoxic insults, such as might occur with transient ischemia or seizures, compromise the endogenous defenses and promote the transition from the early activation pathway to MAC deposition on cortical neurons in vitro (Xiong and McNamara, 2002). The devastating consequences of MAC deposition in cortical gray matter demonstrated here underscore the need to identify small molecule inhibitors selectively targeting the membrane attack pathway, which should reduce or even eliminate the maladaptive consequences of the complement cascade in Alzheimer's disease.

\section{References}

Asghar SS, Pasch MC (2000) Therapeutic inhibition of the complement system. Y2K update. Front Biosci 5:E63-E81.

Benz R, Schmid A, Wiedmer T, Sims PJ (1986) Single-channel analysis of the conductance fluctuations induced in lipid bilayer membranes by complement proteins C5b-9. J Membr Biol 94:37-45.

Berger HJ, Taratuska A, Smith TW, Halperin JA (1993) Activated complement directly modifies the performance of isolated heart muscle cells from guinea pig and rat. Am J Physiol 265:H267-H272.

Bradt BM, Kolb WP, Cooper NR (1998) Complement-dependent proinflammatory properties of the Alzheimer's disease $\beta$-peptide. J Exp Med 188:431-438.

Compston DA, Morgan BP, Campbell AK, Wilkins P, Cole G, Thomas ND, Jasani B (1989) Immunocytochemical localization of the terminal complement complex in multiple sclerosis. Neuropathol Appl Neurobiol 15:307-316.

Davoust N, Nataf S, Reiman R, Holers MV, Campbell IL, Barnum SR (1999) Central nervous system-targeted expression of the complement inhibitor sCrry prevents experimental allergic encephalomyelitis. J Immunol 163:6551-6556.

Halperin JA, Taratuska A, Rynkiewicz M, Nicholson-Weller A (1993) Transient changes in erythrocyte membrane permeability are induced by sublytic amounts of the complement membrane attack complex (C5b-9). Blood 81:200-205

Hardy J, Selkoe DJ (2002) The amyloid hypothesis of Alzheimer's disease: progress and problems on the road to therapeutics. Science 297:353-356.

Itagaki S, Akiyama H, Saito H, McGeer PL (1994) Ultrastructural localization of complement membrane attack complex (MAC)-like immunoreactivity in brains of patients with Alzheimer's disease. Brain Res 645:78-84.

Jackson MB, Stephens CL, Lecar H (1981) Single channel currents induced by complement in antibody-coated cell membranes. Proc Natl Acad Sci USA 78:6421-6425.

Jiang H, Burdick D, Glabe CG, Cotman CW, Tenner AJ (1994) $\beta$-amyloid activates complement by binding to a specific region of the collagen-like domain of the C1q A chain. J Immunol 152:5050-5059.

Liszewski MK, Farries TC, Lublin DM, Rooney IA, Atkinson JP (1996) Control of the complement system. Adv Immunol 61:201-283.
Matis LA, Rollins SA (1995) Complement-specific antibodies: designing novel anti-inflammatories. Nat Med 1:839-842.

McGeer PL, Akiyama H, Itagaki S, McGeer EG (1989) Activation of the classical complement pathway in brain tissue of Alzheimer patients. Neurosci Lett 107:341-346.

Morgan BP (1994) Clinical complementology: recent progress and future trends. Eur J Clin Invest 24:219-228.

Morgan BP (1995) Physiology and pathophysiology of complement: progress and trends. Crit Rev Clin Lab Sci 32:265-298.

Morgan BP (1999) Regulation of the complement membrane attack pathway. Crit Rev Immunol 19:173-198. arsid15078276

Morgan BP, Dankert JR, Esser AF (1987) Recovery of human neutrophils from complement attack: removal of the membrane attack complex by endocytosis and exocytosis. J Immunol 138:246-253. arsid15078276

Paxinos G, Watson C (1982) The rat brain in stereotaxic coordinates. Sydney: Academic.

Racine RJ (1972) Modification of seizure activity by electrical stimulation. II. Motor seizure. Electroencephalogr Clin Neurophysiol 32:281-294.

Schmued LC, Hopkins KJ (2000) Fluoro-Jade B: a high affinity fluorescent marker for the localization of neuronal degeneration. Brain Res 874:123-130.

Scolding NJ, Morgan BP, Houston WA, Linington C, Campbell AK, Compston DA (1989) Vesicular removal by oligodendrocytes of membrane attack complexes formed by activated complement. Nature 339:620-622.

Singhrao SK, Neal JW, Morgan BP, Gasque P (1999) Increased complement biosynthesis by microglia and complement activation on neurons in Huntington's disease. Exp Neurol 159:362-376.

Stein JM, Luzio JP (1991) Ectocytosis caused by sublytic autologous complement attack on human neutrophils: the sorting of endogenous plasmamembrane proteins and lipids into shed vesicles. Biochem J 274:381-386.

Walport MJ (2001) Complement. N Engl J Med 344:1058-1066.

Webster S, Lue LF, Brachova L, Tenner AJ, McGeer PL, Terai K, Walker DG, Bradt B, Cooper NR, Rogers J (1997) Molecular and cellular characterization of the membrane attack complex, C5b-9, in Alzheimer's disease. Neurobiol Aging 18:415-421. arsid8080185

Whitney KD, McNamara JO (2000) GluR3 autoantibodies destroy neural cells in a complement-dependent manner modulated by complement regulatory proteins. J Neurosci 20:7307-7316. arsid8080185

Whitney KD, Andrews PI, McNamara JO (1999) Immunoglobulin G and complement immunoreactivity in the cerebral cortex of Rasmussen's encephalitis patients. Neurology 53:699-708. arsid15078276

Wiedmer T, Sims PJ (1985) Cyanine dye fluorescence used to measure membrane potential changes due to the assembly of complement proteins C5b-9. J Memb Biol 84:249-258.

Wyss-Coray T, Yan F, Lin AH, Lambris JD, Alexander JJ, Quigg RJ, Masliah E (2002) Prominent neurodegeneration and increased plaque formation in complement-inhibited Alzheimer's mice. Proc Natl Acad Sci USA 99:10837-10842.

Xiong Z-Q, McNamara JO (2002) Fleeting activation of ionotropic glutamate receptors sensitizes cortical neurons to complement attack. Neuron 36:363-374.

Yasojima K, Schwab C, McGeer EG, McGeer PL (1999) Up-regulated production and activation of the complement system in Alzheimer's disease brain. Am J Pathol 154:927-936.

Yasuhara O, Aimi Y, McGeer EG, McGeer PL (1994) Expression of the complement membrane attack complex and its inhibitors in Pick disease brain. Brain Res 652:346-349. 